\title{
A Comparative Study of In- and Post-Source Decays of Peptide and Preformed Ions in Matrix-Assisted Laser Desorption Ionization Time-of-Flight Mass Spectrometry: Effective Temperature and Matrix Effect
}

\author{
So Hee Yoon, ${ }^{\text {a Jeong Hee Moon, }}{ }^{\mathrm{b}}$ and Myung Soo Kim ${ }^{\mathrm{a}}$ \\ ${ }^{a}$ Department of Chemistry, Seoul National University, Seoul, Korea \\ ${ }^{\mathrm{b}}$ Medical Proteomics Research Center, KRIBB, Daejeon, Korea
}

In-source decay (ISD) and post-source decay (PSD) of a peptide ion $\left(\left[\mathrm{Y}_{6}+\mathrm{H}\right]^{+}\right)$and a preformed ion (benzyltriphenylphosphonium, BTPP) generated by matrix-assisted laser desorption ionization (MALDI) were investigated with time-of-flight mass spectrometry. $\alpha$-Cyano-4-hydroxycinammic acid (CHCA) and 2,5-dihydroxybenzoic acid (DHB) were used as matrices. For both ions, ISD yield was unaffected by delay time, indicating rapid termination of ISD. This was taken as evidence for rapid expansion cooling of hot "early" plume formed in MALDI. CHCA was hotter than DHB for $\left[\mathrm{Y}_{6}+\mathrm{H}^{+}\right.$while the matrix effect was insignificant for BTPP. The "early" plume temperature estimated utilizing previous kinetic results was $800-900 \mathrm{~K}$, versus $400-500 \mathrm{~K}$ for "late" plume. The results support our previous finding that the temperature of peptide ions interrogated by tandem mass spectrometry was lower than most rough estimates of MALDI temperature. (J Am Soc Mass Spectrom 2010, 21, 1876-1883) @ 2010 Published by Elsevier Inc. on behalf of American Society for Mass Spectrometry

\section{$\mathrm{E}$} ven though matrix-assisted laser desorption ionization (MALDI) [1-5] is widely used to generate gas-phase ions from biomolecules, details of the processes involved are not firmly understood. Outstanding problems include the internal energy of ions formed by MALDI and how it is affected by matrix [2-5]. With the assumption of quasi-thermal equilibrium, the first problem becomes equivalent to the question on effective temperature [6-11]. In post-source decay (PSD) [12] of peptide ions generated by MALDI with two common matrices, $\alpha$-cyano-4-hydroxycinammic acid (CHCA) and 2,5-dihydroxybenzoic acid (DHB), the relative product ion yield is in the order DHB $<$ CHCA $[7,13]$, suggesting the same order for the temperature. Accordingly, CHCA and DHB are often called "hot" and "cold" matrices, respectively. In our recent kinetic studies [14-19] for peptide ions without arginine, which mostly generate $a, b$, and $y$ type product ions, not only relative PSD yield but also photodissociation (PD) rate constant followed the order DHB < CHCA, in agreement with the above classification.

Matrix effect on in-source decay (ISD) of peptide and small protein ions has also been investigated [11, 20-

Address reprint requests to Professor M. S. Kim, Department of Chemistry, Seoul National University, Seoul 151-742, Korea. E-mail: myungsoo@ snu.ac.kr
22]. The main focus of such studies was on the formation of $c$ and $z$ type product ions, which is thought to occur via matrix-to-analyte hydrogen radical transfer [22]. Unlike in PSD, DHB was found to be more efficient than CHCA for such reactions. However, it may be irrelevant to generation of $a, b$, and $y$ ions observed in conventional tandem mass spectrometry, a subject of interest in this work.

Various processes may affect the effective temperature of a peptide ion, such as laser-induced sample explosion, matrix-to-analyte proton transfer, and further cooling of the plume due to its expansion, etc. There have been attempts to study the influence of the processes inherent to matrix-assisted laser desorption (MALD). An example is the photoionization study of neutrals generated by MALD reported by Mowry and Johnston [6]. There, the effective temperature of a neutral was found to be close to matrix sublimation temperature (sublimation temperatures of DHB and CHCA are 443 and $455 \mathrm{~K}$, respectively, according to a recent report by Zenobi et al. [23]). Another was to study dissociation of ions already present inside a solid matrix-analyte mixture, i.e., preformed ions. For example, Vertes et al. [24, 25] investigated ISD of benzylpyridinium and benzyltriphenylphosphonium (BTPP) ions and found that the matrix effect was opposite to the trend observed for peptide ion PSD. In contrast, the 
usual order, DHB < CHCA, was observed in the atmospheric pressure-MALDI study of benzylpyridinium ion carried out by Karas et al. [7].

In our previous dissociation kinetic studies of peptide ions [14-19], we analyzed product ion yields in PSD and time-resolved PD with Rice-RamspergerKassel-Marcus theory [26] and determined critical energy, critical entropy, and temperature. Even though effective temperatures were in the order DHB $<\mathrm{CHCA}$, they were lower than most rough estimates [7-11] of MALDI temperature. Even if hot peptide ions are formed by MALDI, the effective temperature of peptide ions interrogated by PSD and PD can be lower if most of hot ions dissociate inside the source. To see if this was an appropriate explanation for rather low peptide ion temperature determined in our works, a comparative study was made, i.e., ISD versus PSD, preformed ions versus peptide ions, and DHB versus CHCA. Results are reported in this paper, which strongly support our previous kinetic measurements.

\section{Experimental}

\section{Mass Spectrometer}

As shown in Supplemental Information, which can be found in the electronic version of this article, the instrument [14] consists of a MALDI source with delayed extraction, a linear TOF analyzer, an ion gate, and a second-stage TOF analyzer equipped with a reflectron. Due to a deflection system [27], only product ions formed between the exit of this system and the reflectron entrance are detected in PSD. A $337 \mathrm{~nm}$ output from a nitrogen laser (MNL100; Lasertechnik Berlin, Berlin, Germany) was used for MALDI; $20 \mathrm{kV} \mathrm{DC}$ and $1.5 \mathrm{kV}$ AC pulse were used for delayed extraction/ acceleration and the final electrode of the reflectron was kept at $25 \mathrm{kV}$. A commercial MALDI-TOF (Axima CFRplus; Shimadzu, Kyoto, Japan) was also used to record MALDI spectra. Each MALDI spectrum was averaged over 250 MALDI shots and PSD spectrum over 3000 shots on many spots. Three or more duplicate measurements were made to estimate error limits.

\section{Detector Gain Calibration and Ion Intensity Measurement}

The method to calibrate the gain of the MCP detector was reported previously [15]. The instrument was run under the single ion condition. Charge due to each single ion was measured in time-of-flight spectrum, not in mass spectrum, by integrating over a single ion current pulse and averaged over several thousand pulses. For each ion, intensity was measured only for the lowest mass isotopic peak. Then, calculated abundances of higher mass isotopic ions were used to evaluate its total intensity.

\section{Determination of the Threshold Laser Pulse Energy} for MALDI

Throughout the experiment, laser spot size at the sample was fixed ( $5 \mathrm{~mm}$ diameter beam focused by $\mathrm{f}=250$ $\mathrm{mm}$ lens). Instead of the fluence of a laser pulse (pulse energy $\div$ spot area), we will just quote pulse energy to avoid any confusion arising from possibly erroneous measurement of the spot size. We defined the threshold as the condition at which $50 \%$ of the single-shot spectra displayed molecular ion peaks. The threshold determined based on this definition was the same as that based on the definition proposed by Hillenkamp and coworkers [28, 29] because of the excellent gain of the dual MCP installed in the present instrument.

\section{Samples}

Benzyltriphenylphosphonium (BTPP) chloride, YYYYYY $\left(\mathrm{Y}_{6}\right), \mathrm{CHCA}, \mathrm{DHB}$, and other chemicals were purchased from Sigma (St. Louis, MO, USA). A matrix solution prepared with 1:1 acetonitrile/water and 1:1 acetonitrile $/ 0.1 \%$ trifluoroacetic acid was mixed with aqueous solution of BTPP chloride and $\mathrm{Y}_{6}$, respectively. Matrixto-analyte molar ratios for BTPP chloride and $\mathrm{Y}_{6}$ were 20000:1 and 1000:1, respectively.

\section{Results}

Peptide ion, $\left[Y_{6}+H\right]^{+}$

MALDI and PSD spectra of $\left[\mathrm{Y}_{6}+\mathrm{H}\right]^{+}$generated with CHCA and DHB are shown in Figure 1. Product ions appearing in PSD, i.e., $a_{n}(n=2,4,5), b_{n}(n=2-5), y_{n}$ $(n=2-5)$, and $Y$, were assigned previously [15]. Same product ions appear in MALDI spectra also. In particular, $c$ and $z$ product ions are absent. Product ion intensity sums in ISD and PSD divided by the precursor ion intensity, to be called YISD and YPSD, respectively, were measured from the spectra.

Matrix, laser pulse energy, and the time between laser irradiation and ion extraction (delay time) can affect product ion yields measured with delayed extraction MALDI-TOF. We investigated the influence of delay time by varying it over 150-500 ns. A change in delay time without other adjustment led to a change in resolution and hence peak heights. Regardless, peak intensity measured by the present method was hardly affected by instrument tuning. Delay time dependences for YISD and YPSD with CHCA measured over 150-500 ns are shown in Figure $2 a$ and $b$, respectively. Neither YISD nor YPSD for $\left[\mathrm{Y}_{6}+\mathrm{H}\right]^{+}$is affected by delay time within the error limits. Same trend was observed regardless of laser pulse energy. Even though YISD and YPSD obtained with DHB were much smaller, general trend was the same.

We limited the laser pulse energy to 1-2 times the threshold, which is the range used in analytical application of MALDI and in our kinetic study. The thresh- 


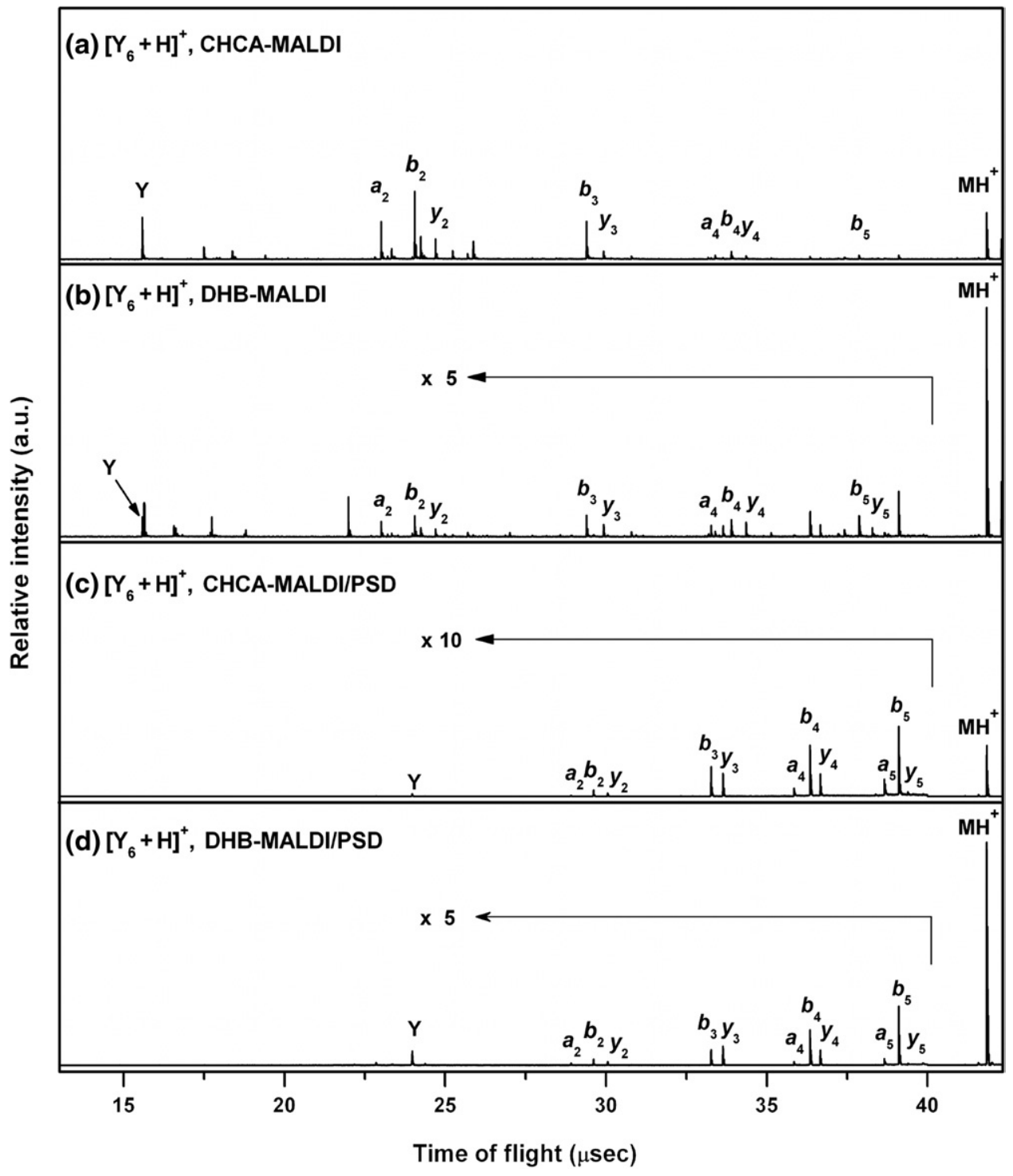

Figure 1. MALDI and PSD spectra of $\left[\mathrm{Y}_{6}+\mathrm{H}^{+}\right.$. (a) CHCA-MALDI, (b) DHB-MALDI, (c) CHCA-MALDI/PSD, and (d) DHB-MALDI/PSD. Pulse energies used in CHCA- and DHB-MALDI were 1.5 and $2.5 \mu \mathrm{J} /$ pulse, respectively. Spectra are drawn such that relative intensities of $\left[\mathrm{Y}_{6}+\mathrm{H}\right]^{+}$ with CHCA and DHB are the same as actually measured.

old pulse energies for $\left[\mathrm{Y}_{6}+\mathrm{H}\right]^{+}$with CHCA and DHB were 1.1 and $1.8 \mu \mathrm{J} /$ pulse, respectively. Since the amount of laser energy transferred is also affected by absorbance of the matrix, it may be better to compare YISD and YPSD at the same relative pulse energy, i.e., pulse energy divided by the threshold value. YISD and YPSD versus relative pulse energy are shown in Figure 3. It is to be noted that product ions appeared in MALDI and PSD spectra even at the threshold. YISD increased rapidly with pulse energy, more rapidly with CHCA than with DHB. YPSD tended to increase also, but slowly. Even though PSD spectral patterns with CHCA and DHB were similar, YPSD with CHCA was around four times larger than with $\mathrm{DHB}$, as found in our previous works [15].
Preformed Ion, BTPP

DHB-MALDI and PSD spectra of BTPP $(m / z$ 353.1) are shown in Figure 4. Corresponding spectra obtained with CHCA were similar. $\mathrm{C}_{7} \mathrm{H}_{7}^{+}(\mathrm{m} / \mathrm{z}$ 91.1) and $\mathrm{P}\left(\mathrm{C}_{6} \mathrm{H}_{5}\right)_{3}^{+}(\mathrm{m} / \mathrm{z}$ 262.1) were major product ions in MALDI [25]. $\mathrm{P}\left(\mathrm{C}_{6} \mathrm{H}_{4}\right)_{2}^{+}(\mathrm{m} / z$ 183.0) also appeared in PSD.

As for $\left[\mathrm{Y}_{6}+\mathrm{H}\right]^{+}$, dissociation yields of BTPP were hardly affected by delay time as demonstrated in Figure $2 c$ for ISD with CHCA-MALDI. The same trend was observed regardless of laser pulse energy and matrix, and also observed for YPSD.

In MALDI of BTPP with CHCA and DHB, the threshold laser pulse energies were around 0.85 and 1.9 $\mu \mathrm{J} /$ pulse, respectively. YISD and YPSD versus relative 

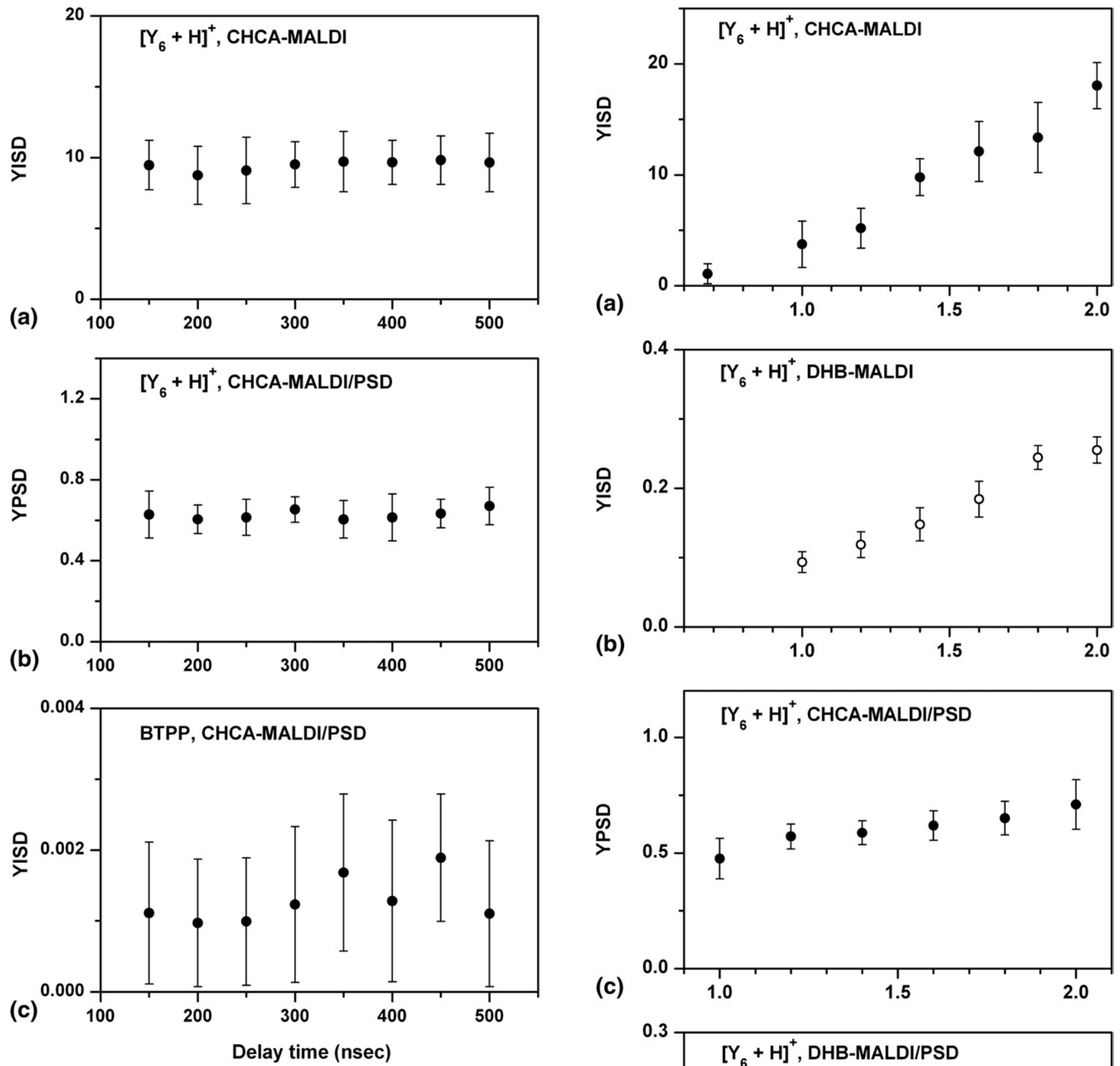

Figure 2. Product ion yields measured as a function of delay time ( \pm 20 nsec accuracy). (a) YISD for $\left[\mathrm{Y}_{6}+\mathrm{H}\right]^{+}$with CHCA at $1.5 \mu \mathrm{J} /$ pulse. (b) YPSD for $\left[\mathrm{Y}_{6}+\mathrm{H}^{+}\right]^{+}$with CHCA at $1.5 \mu \mathrm{J} /$ pulse. (c) YISD for BTPP with CHCA at $1.2 \mu \mathrm{J} /$ pulse.

pulse energy are shown in Figure 5. Unlike for $\left[\mathrm{Y}_{6}+\right.$ $\mathrm{H}]^{+}$, YISD values, and YPSD also, measured with DHB and CHCA were comparable and were independent of the laser pulse energy, even though intensities of both precursor and product ions increased rapidly. Also, YISD measured in this work was smaller than previous results [25] obtained with continuous extraction linear TOF by orders of magnitude. Smaller YISD with delayed extraction reflectron TOF is unexpected because product ions formed some time after laser irradiation will not be counted as signal [7] in continuous extraction while those formed outside the ion source will be counted as the precursor ion signal in linear TOF. We
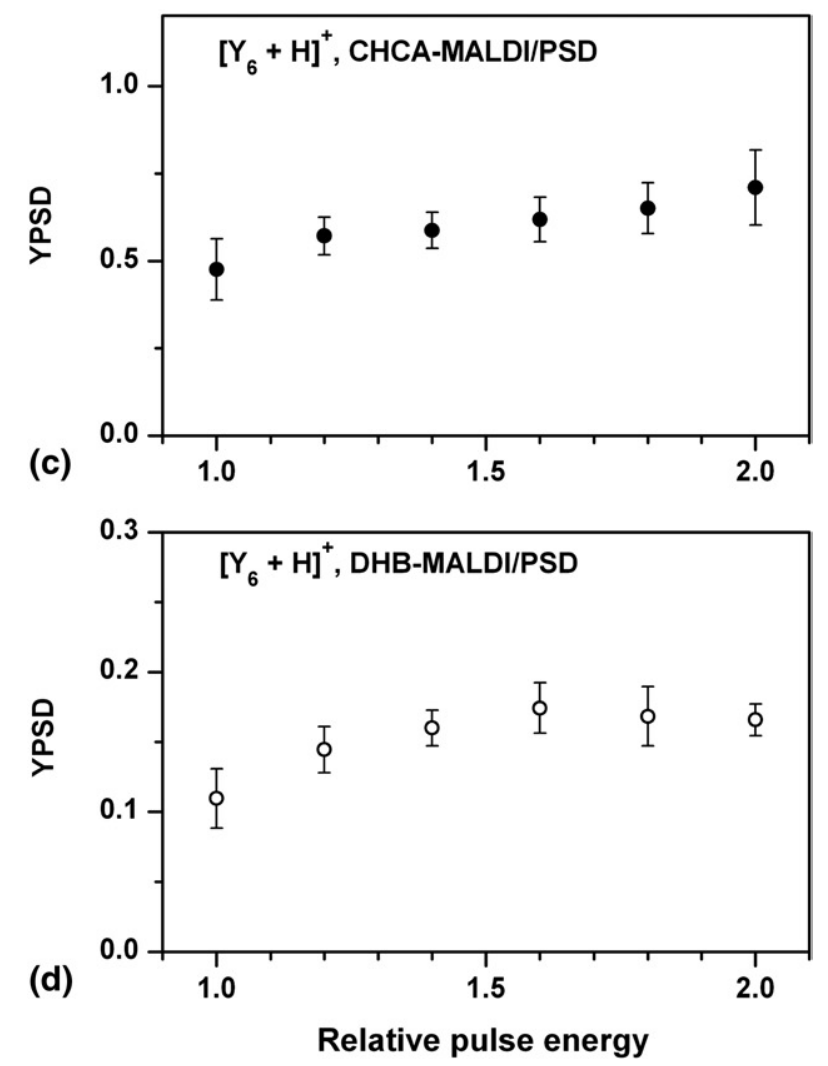

Figure 3. Laser pulse energy dependence of product ion yields for $\left[\mathrm{Y}_{6}+\mathrm{H}\right]^{+}$. (a) YISD with CHCA, (b) YISD with DHB, (c) YPSD with CHCA, and (d) YPSD with DHB. Abscissa is the relative pulse energy, i.e., pulse energy divided by the threshold value. The thresholds with CHCA and DHB are 1.1 and $1.8 \mu \mathrm{J} /$ pulse, respectively. YISDs measured below the thresholds are also drawn. 


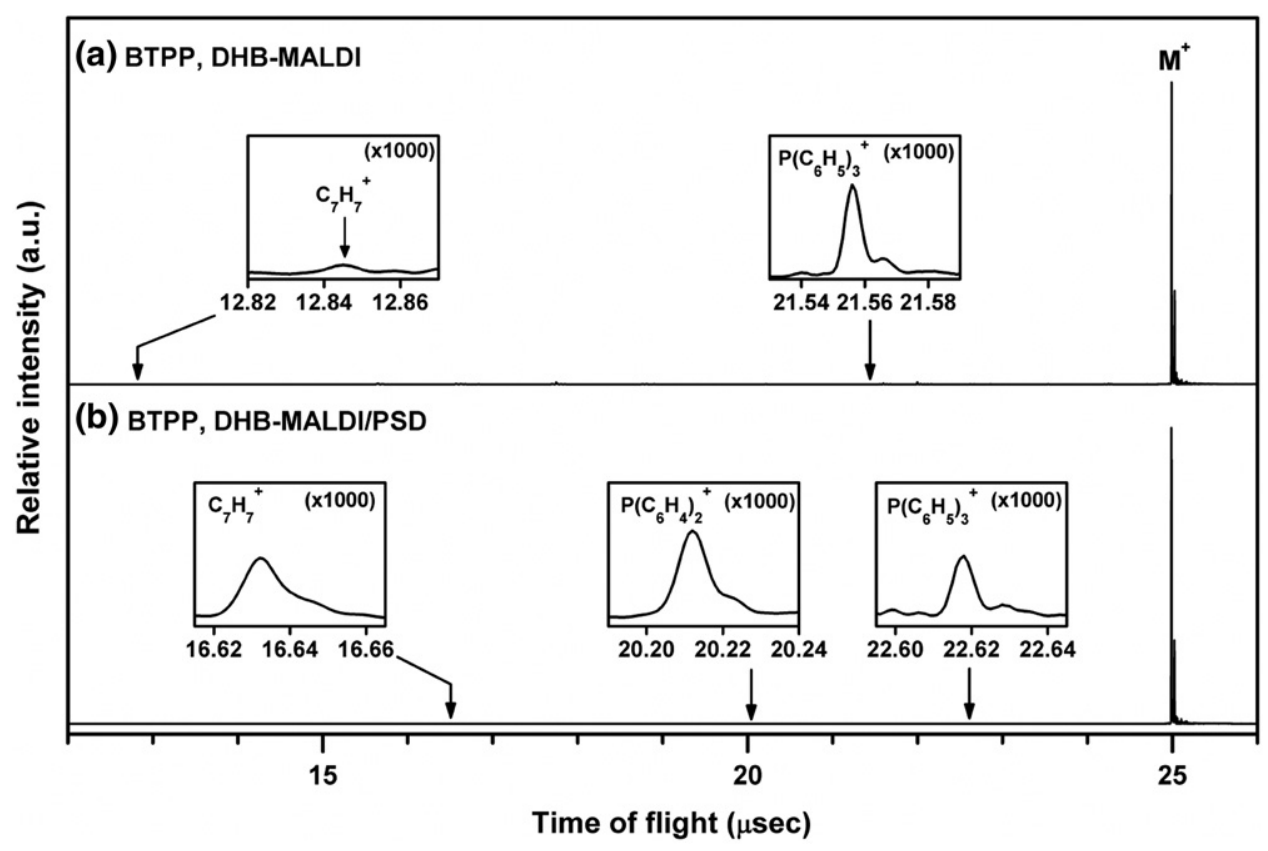

Figure 4. (a) MALDI and (b) PSD spectra of BTPP obtained with DHB-MALDI. Unlike in reference [25], fragment ions are very weak as shown in insets.

speculate that the difference between the two works might have arisen due to different methods for intensity measurement. Extremely weak ISD signals in MALDI of BTPP were also confirmed through measurements with a commercial instrument (Axima CFRplus; Shimadzu).

\section{Discussion}

The main purpose of this work was to find an explanation for lower temperature $(400-470 \mathrm{~K})$ of peptide ions determined in our tandem mass spectrometric study [16] than previous rough estimates for MALDI temperature such as $800-1100 \mathrm{~K}$ reported by Karas et al. [7, 8] and $740-960 \mathrm{~K}$ reported by Yergey et al. [11]. If a single effective temperature represents the internal energy distribution for a precursor ion promptly formed by MALDI and if this temperature is maintained throughout the delay period, YISD will increase almost in proportion to delay time. In this regard, the single most important observation made in this work is that YISDs for $\left[\mathrm{Y}_{6}+\mathrm{H}\right]^{+}$and BTPP were unaffected by delay time. This indicates that ISD subsides very rapidly, within the minimum delay time (150 ns) used, and that the temperature for precursors to PSD is lower than that to ISD. Even though delay time independence was also observed for ISD of peptide ions to $c$ and $z$ type ions [20-22], it may not be directly relevant to the subject of interest in this work as mentioned in the introductory section.

In the delayed extraction linear TOF study of benzylpyridium derivatives, Karas et al. [7] noted that two thermally different populations of precursor ions had to be postulated to explain overall results. Two models were proposed, i.e., very rapid cooling of the initially hot plume and bimodal energy distribution for "prompt" precursor ions. Delay time independence of YISD observed here may be explained similarly, as follows. In model 1, a hot plume formed upon laserinduced sample explosion expands and cools rapidly. ISD occurring efficiently in the early plume subsides rapidly due to expansion cooling and becomes insignificant by the time extraction begins. Slow dissociation of cooled precursor ions can be detected in PSD because of its longer observation time scale (around $10 \mu \mathrm{s}$ ). Instead of the coexistence of two different thermal populations of ions in a same plume, it may be more reasonable to postulate two different ion formation mechanisms [11]. Hence, in our model 2, cooler ions such as those desorbed from cooled clusters contribute to PSD. In both models, precursor ions subjected to tandem mass spectrometric interrogation will be cooler than those contributing to ISD.

Unlike in a previous report [25], YISDs for BTPP generated with CHCA and DHB were similar, suggesting similar temperatures for early DHB and CHCA plumes. Then, similar extent of expansion cooling of the two plumes might result in similar YPSD as observed. However, it will be shown later that temperatures for early DHB and CHCA plumes are somewhat different. Very small YISD and YPSD of BTPP suggest that its dissociation occurs at the very high end of the internal energy distribution where thermal equilibrium may not be an accurate description. Then, BTPP may not be useful as a thermometer ion for the study of MALDI process.

Compared with BTPP, product ions are much more intense, by orders of magnitudes, in MALDI spectra of $\left[\mathrm{Y}_{6}+\mathrm{H}\right]^{+}$. Very efficient ISD indicates small critical 

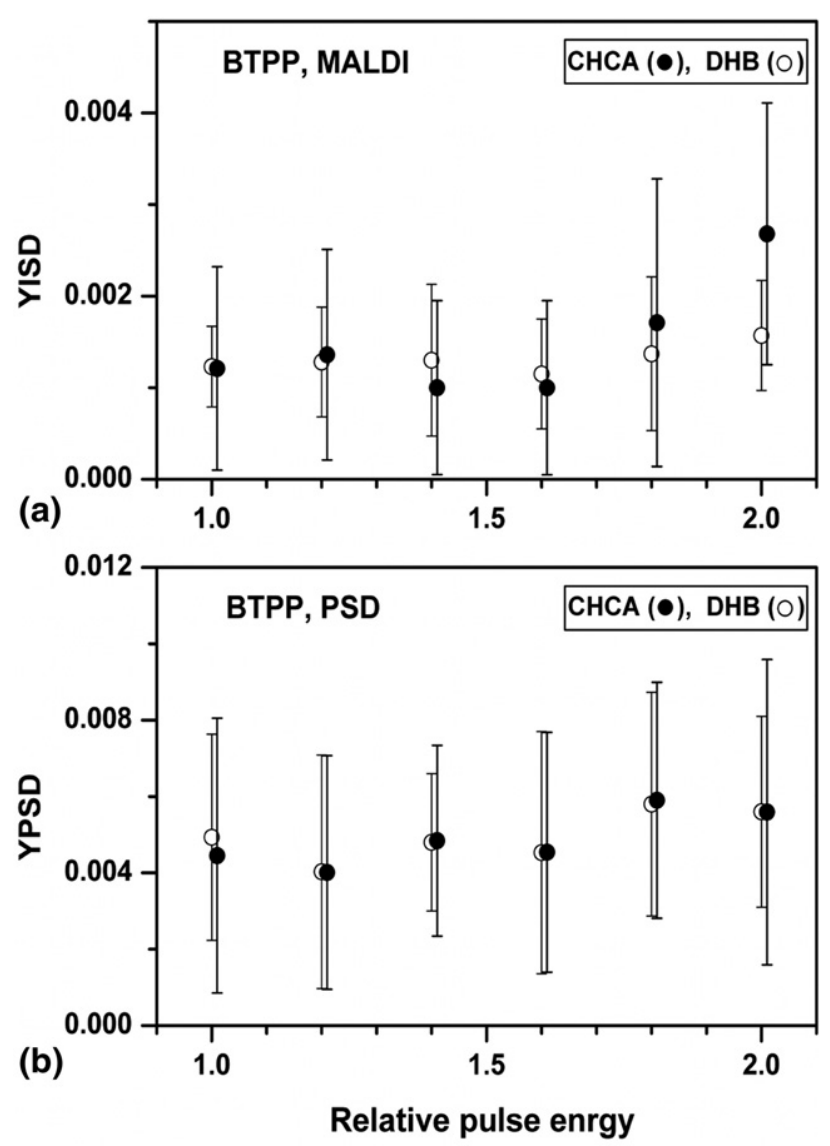

Figure 5. Laser pulse energy dependence of product ion yields for BTPP. (a) YISD and (b) YPSD. Data obtained with CHCA and DHB are shown as dark (filled circles) and open (open circles), respectively. Abscissa is the relative pulse energy, i.e., pulse energy divided by the threshold value. The thresholds with CHCA and DHB are 0.85 and $1.9 \mu \mathrm{J} /$ pulse, respectively.

energy for the dissociation of $\left[\mathrm{Y}_{6}+\mathrm{H}\right]^{+}$, certainly much smaller than for the $\mathrm{P}-\mathrm{C}$ bond cleavage in BTPP. Critical energy for $\left[\mathrm{Y}_{6}+\mathrm{H}\right]^{+}$determined in our previous study was $0.60 \mathrm{eV}$ [16], much smaller than usual single bond energy. The fact that substantial fractions of $\left[\mathrm{Y}_{6}+\mathrm{H}\right]^{+}$ undergo dissociation makes it reasonable to analyze the results under the thermal assumption. In this regard, it is to be noted that matrix effect in $\left[\mathrm{Y}_{6}+\mathrm{H}\right]^{+}$dissociation is significant unlike in BTPP. To reiterate data in the previous section, YPSD with CHCA is larger than with DHB by a factor of 4 and YISD by nearly two orders of magnitude. Part of the matrix effect on measured YISD values arises because larger fraction of $\left[\mathrm{Y}_{6}+\mathrm{H}\right]^{+}$generated with $\mathrm{CHCA}$ undergoes dissociation in the region between the ion source and the detector than with DHB. This results in smaller precursor ion intensity with CHCA, which is used as the denominator in YISD. We attempted to estimate the extent of the post-source depletion of precursor ion as follows. In PSD, we observed dissociation occurring between the exit of the deflection system and the entrance of the reflection, which corresponded to the time range of 10.3-17.1 $\mu$ s after laser irradiation (see Supplemental
Information). This PSD time range was $16.5 \%$ of the total flight time $(41.1 \mu \mathrm{s})$ of the precursor ion. Then, roughly speaking, around six times more dissociation must have occurred than observed by PSD. Let us take 0.60 and 0.15 as typical YPSD with CHCA and DHB, respectively. Then, if the precursor ion did not dissociate after the source, its intensity would have been larger by $4.6(0.60 \times 6+1)$ in measurement with CHCA. Corresponding value with DHB was 1.9. Even after this correction, YISD with CHCA was still larger than with DHB by a factor 30 . We also realized that the usual guideline for threshold determination might be inappropriate for ions undergoing efficient dissociation even at the threshold. That is, it would be more appropriate to count the appearance of any ionic species from the peptide, either $\left[\mathrm{Y}_{6}+\mathrm{H}\right]^{+}$or its products, rather than $\left[\mathrm{Y}_{6}+\mathrm{H}\right]^{+}$only. The new definition resulted in lower thresholds, 0.75 and $1.6 \mu \mathrm{J} /$ pulse with CHCA and DHB, respectively. YISD values measured at the new thresholds were 1.1 and 0.089 with CHCA and DHB, respectively, a factor of 12 difference. After correcting for the post-source dissociation, difference further narrowed, YISD with CHCA being larger by a factor of 5 than with $\mathrm{DHB}$, getting close to the matrix effect in PSD. That is, even though ISD with CHCA looks dramatically efficient, part of it is an illusion caused by efficient dissociation of $\left[\mathrm{Y}_{6}+\mathrm{H}\right]^{+}$. Since the peptide ion signal at the threshold of CHCA-MALDI is very weak, one would increase pulse energy, which is not very effective due to dissociation. Eventually, one ends up using very large pulse energy, generating even hotter ions. After corrections, however, the fact still remains that $\mathrm{CHCA}$ is hotter than DHB.

The fact that $\left[\mathrm{Y}_{6}+\mathrm{H}\right]^{+}$generated with $\mathrm{CHCA}$ is hotter than that with DHB may have something to do with the ionization step. If $\left[\mathrm{Y}_{6}+\mathrm{H}\right]^{+}$is formed by proton transfer between $Y_{6}$ and protonated matrix, a matrix with smaller proton affinity (PA) will form hotter $\left[\mathrm{Y}_{6}+\mathrm{H}\right]^{+}$. Previously $[15,16]$, we noted that the experimental temperature order of $\mathrm{DHB}<$ sinapinic acid (SA) $<$ CHCA did not correlate with the PA order $\mathrm{CHCA} \approx \mathrm{DHB}<\mathrm{SA}$ in the literature [5], suggesting that PA is not an important factor affecting peptide ion temperature. Then, the matrix effect in $\left[\mathrm{Y}_{6}+\mathrm{H}\right]^{+}$may be prominent simply because CHCA plume is a little hotter than that of DHB. $\left[\mathrm{Y}_{6}+\mathrm{H}\right]^{+}$will be sensitive to small difference in plume temperature because of its small dissociation critical energy while BTPP will not be as sensitive. CHCA may be hotter than DHB because its sublimation temperature is higher. That is, relatively larger pulse energy needed with CHCA results in hotter plume. Zenobi et al. [23] measured sublimation temperature of several matrices (409-455 K), investigated its influence on ion internal energy, and found no correlation between the two. Regardless, we speculate that sublimation temperature might play some role, considering that glycerol infrared MALDI [30,31] is softer than UV-MALDI. The sublimation temperature of glycerol is $380 \mathrm{~K}$ when the guideline suggested by Zenobi et 
al. [23] is applied to its vapor pressure data [32]. Further investigations are needed to find out how matrices affect the plume and peptide ion temperatures, such as acquisition of more accurate data for matrix sublimation temperature.

Rough estimation of the early plume temperature from YISD can be helpful to compare with MALDI temperatures in literature [6-11]. Temperature of $\left[Y_{6}+\right.$ $\mathrm{H}]^{+}$under tandem mass spectrometric interrogation, or "late" plume temperature, determined in our previous kinetic studies [16] was around 470 and $400 \mathrm{~K}$, respectively, with CHCA and DHB. The rate-energy relation, $k_{\text {tot }}(E)$, calculated with the critical energy $(0.60 \mathrm{eV})$ and entropy $\left(-28.4 \mathrm{eu}\right.$ with $\left.1 \mathrm{eu}=4.184 \mathrm{~J} \mathrm{~K}^{-1} \mathrm{~mol}^{-1}\right)$ determined previously is drawn in Figure 6 together with the internal energy distributions at the above temperatures. Let us assume that ISD occurs within 50 nsec after laser irradiation, corresponding to the minimum rate constant of $1.4 \times 10^{7} \mathrm{sec}^{-1}$. The minimum energy needed for ISD read from the $k_{\text {tot }}(E)$ curve is 11.7 $\mathrm{eV}$. Then, let us assume that the temperature difference between "early" CHCA and DHB plumes is the same as that between "late" plumes and that roughly around 80 and $20 \%$ of $\left[\mathrm{Y}_{6}+\mathrm{H}\right]^{+}$undergo ISD with CHCA and $\mathrm{DHB}$, respectively. The "early plume" temperature thus

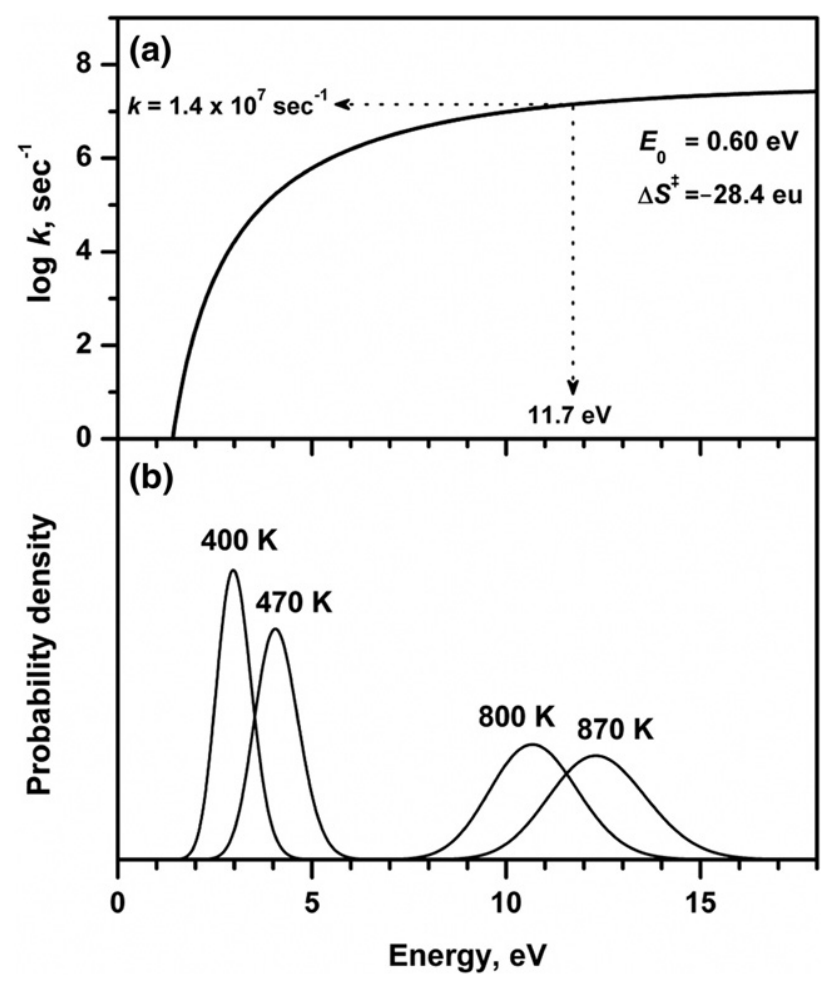

Figure 6. (a) $k_{\mathrm{tot}}(E)$ calculated with $E_{0}=0.60 \mathrm{eV}, \Delta S^{\ddagger}=-28.4 \mathrm{eu}$ reported in reference [1]. Internal energy of $11.7 \mathrm{eV}$ needed for $k_{\text {tot }}$ of $1.4 \times 10^{7} \mathrm{~s}^{-1}$ is marked. (b) Internal energy distributions of $\left[\mathrm{Y}_{6}+\right.$ $\mathrm{H}]^{+}$at $T=400,470,800$, and $870 \mathrm{~K} ; 400$ and $470 \mathrm{~K}$ are the effective temperatures for ions contributing to PSD in DHB- and CHCAMALDI, respectively, determined previously [16]; 800 and $870 \mathrm{~K}$ are those contributing to ISD in DHB- and CHCA-MALDI, respectively, estimated in this work. estimated was around 870 and $800 \mathrm{~K}$ for CHCA and $\mathrm{DHB}$, respectively. The internal energy distributions at these temperatures are also drawn in Figure 6. The results seem to be in fair agreement with previous rough estimates for "early" plume temperature such as 740-960 K with CHCA reported by Yergey et al. [11]. Also to be quoted are the results from a recent molecular dynamics simulation study of DHB-MALDI carried out by Knochenmuss and Zhigilei [10]. There, "early" DHB plume with temperature $800 \mathrm{~K}$ was formed by MALDI and underwent expansion cooling to about half that value, almost in exact agreement with the present results.

Even though the time span for ISD (tens of nanosecond) was much shorter than that for PSD (several microseconds), ISD of peptide ions was more efficient than PSD. Hence, there is no doubt that peptide ions undergoing PSD are much cooler than those contributing to ISD regardless of the model used, i.e., expansion cooling. This strongly supports our previous finding that temperature for peptide ions under tandem mass spectrometric interrogation are rather low. In this regard, we would like to mention that $1090 \mathrm{~K}$ or higher temperature was found for peptide ions under tandem mass spectrometric interrogation when our experimental PD rate constants for [YGGFL $+\mathrm{H}^{+}$[19] were analyzed with $k_{\mathrm{tot}}(E)$ s determined by other methods [33, 34]. Further calculations with the same $k_{\text {tot }}(E)$ s showed very rapid dissociation of hot ions such that acquisition of MALDI spectra for peptides should have been virtually impossible, completely against the reality.

\section{Conclusion}

We have found that ISD occurs rapidly from hot precursor ions in "early" plume and terminates well before extraction begins while PSD occurs slowly from cooler ions surviving ISD. Expansion cooling of hot "early" plume seems to be responsible for rapid termination of ISD. Cooler precursor ions might be formed by desorption from clusters in "late" plume. However, clusters themselves should have been cooled by expansion. Otherwise, hot desorbed ions would dissociate rapidly also. We suggest that $\left[\mathrm{Y}_{6}+\mathrm{H}\right]^{+}$at $800-900 \mathrm{~K}$ in "early" plume undergoes expansion cooling to $400-500 \mathrm{~K}$ in "late" plume, in agreement with findings in a recent molecular dynamics simulation study [10].

Previously, we determined critical energy and entropy for peptide ion dissociation by analyzing product ion yields in PSD and time-resolved PD [15-19]. Ion temperature determined in the same analysis was lower than previous rough estimates for MALDI temperature. When PSD yield was not included in the analysis, good fits could be achieved by increasing all three parameters simultaneously. Hence, the present finding that "late" plume temperature is certainly much lower than that of "early" plume strongly supports the critical energy and entropy determined by combined PSD/PD analysis, 
both of which were smaller than previous estimates $[33,34]$.

\section{Acknowledgments}

The authors acknowledge financial support for this work by the National Research Foundation, Republic of Korea. S.H.Y. thanks the Ministry of Education, Science, and Technology, Republic of Korea, for Brain Korea 21 Fellowship.

\section{Appendix A Supplementary Material}

Supplementary material associated with this article may be found in the online version at doi:10.1016/ j.jasms.2010.07.001.

\section{References}

1. Hillenkamp, F.; Peter-Katalinić, J. MALDI MS; Wiley-VCH: Weinheim, 2007; p. 1-108.

2. Dreisewerd, K. The Desorption Process in MALDI. Chem. Rev. 2003, 103, 395-425.

3. Karas, M.; Krüger, R. Ion Formation in MALDI: The Cluster Ionization Mechanism. Chem. Rev. 2003, 103, 427-439.

4. Knochenmuss, R.; Zenobi, R. MALDI. Ionization: The Role of In-Plum Processes. Chem. Rev. 2003, 103, 441-452.

5. Knochenmuss, R. Ion Formation Mechanisms in UV-MALDI. Analyst 2006, 131, 966-986.

6. Mowry, C. D.; Johnston, M. V. Internal Energy of Neutral Molecules Ejected by Matrix-Assisted Laser Desorption. J. Phys. Chem. 1994, 98, 1904-1909.

7. Gabelica, V.; Schulz, E.; Karas, M. Internal Energy Build-Up in MatrixAssisted Laser Desorption/Ionization. J. Mass Spectrom. 2004, 39, 579593.

8. Schulz, E.; Karas, M.; Rosu, F.; Gabelica, V. Influence of the Matrix on Analyte Fragmentation in Atmospheric Pressure MALDI. J. Am. Soc. Mass Spectrom. 2006, 17, 1005-1013.

9. Greisch, J.-F.; Gabelica, V.; Remacle, F.; De Pauw, E. Thermometer Ions for Matrix-Enhanced Laser Desorption/Ionization Internal Energy Calibration. Rapid Commun. Mass Spectrom. 2003, 17, 1847-1854.

10. Knochenmuss, R.; Zhigilei, L. V. Molecular Dynamics Simulations of MALDI: Laser Fluence and Pulse Width Dependence of Plume Characteristics and Consequences for Matrix and Analyte Ionization. J. Mass Spectrom. 2010, 45, 333-346.

11. Campbell, J. M.; Vestal, M. L.; Blank, P. S.; Stein, S. E.; Epstein, J. A.; Yergey, A. L. Fragmentation of Leucine Enkephalin as a Function of Laser Fluence in a MALDI TOF-TOF. J. Am. Soc. Mass Spectrom. 2007, 18, 607-616.

12. Spengler, B. Post-Source Decay Analysis in Matrix-Assisted Laser Desorption/Ionization Mass Spectrometry of Biomolecules. J. Mass Spectrom. 1997, 32, 1019-1036.

13. Stimson, E.; Truong, O.; Richter, W. J.; Waterfield, M. D.; Burlingame, A. L. Enhancement of Charge Remote Fragmentation in Protonated Peptides by High-Energy CID MALDI-TOF-MS Using "Cold" Matrices. Int. J. Mass Spectrom. Ion Processes 1997, 169/170, 231-240.

14. Yoon, S. H.; Kim, M. S. Development of a Time-Resolved Method for Photodissociation Mechanistic Study of Protonated Peptides: Use of a Voltage-Floated Cell in a Tandem Time-of-Flight Mass Spectrometer. J. Am. Soc. Mass Spectrom. 2007, 18, 1729-1739.
15. Moon, J. H.; Yoon, S. H.; Kim. M. S. Temperature of Peptide Ions Generated by Matrix-Assisted Laser Desorption Ionization and Their Dissociation Kinetic Parameters. J. Phys. Chem. B 2009, 113, 2071-2076.

16. Yoon, S. H.; Moon, J. H.; Kim, M. S. Time-Resolved Photodissociation Study of Singly Protonated Peptides with a Histidine Residue Generated by Matrix-Assisted Laser Desorption Ionization: Dissociation Rate Constant and Internal Temperature. J. Am. Soc. Mass Spectrom. 2009, 20, 1522-1529.

17. Yoon, S. H.; Moon, J. H.; Chung, Y. J.; Kim, M. S. Influence of Basic Residue on Dissociation Kinetics and Dynamics of Singly Protonated Peptides: Time-Resolved Photodissociation Study. J. Mass Spectrom. 2009, 44, 1532-1537.

18. Bae, Y. J.; Yoon, S. H.; Moon, J. H.; Kim, M. S. Optimization of Reflectron for Kinetic and Mechanistic Studies with Multiplexed Multiple Tandem $\left(\mathrm{MS}^{\mathrm{n}}\right)$ Time-of-Flight Mass Spectrometry. Bull. Korean Chem. Soc. 2010, 31, 92-99.

19. Moon, J. H.; Yoon, S. H.; Bae, Y. J.; Kim, M. S. Dissociation Kinetics of Singly Protonated Leucine Enkephalin Investigated by Time-Resolved Photodissociation Tandem Mass Spectrometry. J. Am. Soc. Mass Spectrom. 2010, in press.

20. Brown, R. S.; Feng, J.; Reiber, D. C. Further Studies of In-Source Fragmentation of Peptides in Matrix-Assisted Laser DesorptionIonization. Int. J. Mass Spectrom. Ion Processes 1997, 169/170, 1-18.

21. Takayama, M. In-Source Decay Characteristics of Peptides in MatrixAssisted Desorption/Ionization Time-of-Flight Mass Spectrometry. J. Am. Soc. Mass Spectrom. 2001, 12, 420-427.

22. Köcher, T.; Engström, A.; Zubarev, R. A. Fragmentation of Peptides in MALDI In-Source Decay Mediated by Hydrogen Radicals. Anal. Chem. 2005, 77, 172-177.

23. Stevenson, E.; Breuker, K.; Zenobi, R. Internal Energies of Analyte Ions Generated from Different Matrix-Assisted Laser Desorption/Ionization Matrices. J. Mass Spectrom. 2000, 35, 1035-1041.

24. Luo, G.; Marginean, I.; Vertes, A. Internal Energy of Ions Generated by Matrix-Assisted Laser Desorption/Ionization. Anal. Chem. 2002, 74, 6185-6190.

25. Luo, G.; Marginean, I.; Ye, L.; Vertes, A. Competing Ion Decomposition Channels in Matrix-Assisted Laser Desorption Ionization. J. Phys. Chem. B 2008, 112, 6952-6956.

26. Holbrook, K. A.; Pilling, M. J.; Robinson, S. H. Unimolecular Reactions, Wiley: Chichester, 1996; p. 39-78.

27. Yoon, S. H.; Moon, J. H.; Choi, K. M.; Kim, M. S. A Deflection System to Reduce the Interference from Post-Source Decay Product Ions in Photodissociation Time-of-Flight Mass Spectrometry. Rapid Commun. Mass Spectrom. 2006, 20, 2201-2208.

28. Dreisewerd, K.: Schürenberg, M.; Karas, M.; Hillenkamp, F. Influence of the Laser Intensity and Spot Size on the Desorption of Molecules and Ions in Matrix-Assisted Laser Desorption/Ionization. Int. J. Mass Spectrom. Ion Processes 1995, 141, 127-148.

29. Feldhaus, D.; Menzel, C.; Berkenkamp, S.; Hillenkamp, F.; Dreisewerd K. The Role of the Laser Pulse Duration in Infrared Matrix-Assisted Laser Desorption/Ionization Mass Spectrometry. J. Mass Spectrom. 2000, $35,1320-1328$.

30. Overberg, A.; Karas, M.; Bahr, U.; Kaufmann, R.; Hillenkamp, F. Matrix-Assisted Infrared-Laser $(2.94 \mu \mathrm{m})$ Desorption/Ionization Mass Spectrometry of Large Biomolecules. Rapid Commun. Mass Spectrom. 1990, 4, 293-296.

31. Overberg, A.; Karas, M.; Hillenkamp, F.; Cotter, R. J. Matrix-Assisted Laser Desorption of Large Biomolecules with a TEA-CO ${ }_{2}$-Laser. Rapid Commun. Mass Spectrom. 1991, 5, 128-131.

32. Lide, D. R. CRC Handbook of Chemistry and Physics, 85th ed.; CRC Press: 2004; p. 6-77

33. Schnier, P. D.; Price, W. D.; Strittmatter, E. F.; Williams, E. R. Dissociation Energetics and Mechanisms of Leucine Enkephalin $(\mathrm{M}+\mathrm{H})^{+}$and $(2 \mathrm{M}+\mathrm{X})^{+}$ions $(\mathrm{X}=\mathrm{H}, \mathrm{Li}, \mathrm{Na}, \mathrm{K}$, and $\mathrm{Rb})$ Measured by Blackbody Infrared Radiative Dissociation. J. Am. Soc. Mass Spectrom. 1997, 8 , 771-780.

34. Laskin, J. Energetics and Dynamics of Fragmentation of Protonated Leucine Enkephalin from Time- and Energy-Resolved Surface-Induced Dissociation Studies. J. Phys. Chem. A 2006, 110, 8554-8562. 